\title{
Ocorrência de Cryptosporidium spp. em bezerros bubalinos no estado do Pará
}

\section{Occurrence of Cryptosporidium spp. in calves buffaloes in the state of Pará}

\author{
Karen Cristiane Destro', Rinaldo Batista Viana ${ }^{2 *}$, Raimundo Nonato Moraes Benigno², \\ Luciara Celi da Silva Chaves $^{2}$, Washington Luiz Assunção Pereira ${ }^{2}$
}

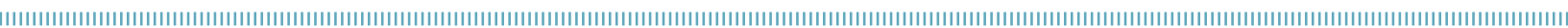

RESUMO: A criptosporidiose é uma zoonose causada por protozoários do gênero Cryptosporidium, que acomete diversas espécies animais, inclusive o homem. Com o presente estudo objetivou-se determinar a ocorrência da infecçáo por Cryptosporidium spp. em bubalinos criados no estado do Pará. Para tanto, foram colhidas fezes de 253 bubalinos, de um dia até seis meses de idade. As fezes colhidas diretamente do reto foram classificadas como diarreicas e não diarreicas segundo a consistência e o aspecto, e analisadas em microscópio óptico após procedimentos de centrifugação, concentraçáo e coloraçáo pelo corante de Kinyoun. Observou-se uma ocorrência de Cryptosporidium spp. em 2,37\% (6/253) das amostras, sendo todas não diarreicas. Não foi estabelecida relação significativa entre a infecção por Cryptosporidium spp., os sinais clínicos de diarreia e a idade dos animais ( $\mathrm{p}>0,05)$. Deste modo, conclui-se que a infecção pelo protozoário Cryptosporidium spp. em bubalinos no estado do Pará apresenta uma baixa ocorrência em animais na faixa etária de um dia até seis meses de idade.

PALAVRAS-CHAVE: búfalos; criptosporidiose; protozoário; diarreia.

\begin{abstract}
Cryptosporidiosis is a zoonosis caused by opportunistic protozoa of the genus Cryptosporidium spp. that attacks various animal species, including humans. This study had the objective of determining the occurrence of infection by Cryptosporidium spp. in buffalo raised in the state of Pará, Brazil, and establishing the relations between Cryptosporidium spp., the age of the animal and the presence of clinical signs of diarrhea. A total of 253 samples of feces from one day to 6-month old buffalo were analyzed. The fecal samples, collected directly from the rectum, were classified as diarrheal and non-diarrheal and analyzed with an optical microscope after centrifuge-concentration and staining with Kinyoun stain. Laboratory analysis revealed the presence of Cryptosporidium spp. in $2.37 \%(6 / 253)$ samples, all of which had a firm consistency. No significant relation was established between infection by Cryptosporidium spp., the animals age from one day to 6-month and clinical symptoms of diarrhea.
\end{abstract}

KEYWORDS: buffalo; cryptosporidiosis; protozoarian; diarrhea.

'Programa de Pós-graduação em Ciência Animal, Núcleo de Ciências Agrárias e Desenvolvimento Rural, Universidade Federal do Pará (UFPA). Campus Universitário do Guamá - Belém (PA), Brasil

${ }^{2}$ Programa de Pós-graduação em Saúde e Produção Animal na Amazônia, Instituto da Saúde e Produção Animal, Universidade Federal Rural da Amazônia (Ufra) - Belém (PA), Brasil

*Autor correspondente: rinaldovianna@hotmail.com

Recebido em: 21/07/2012. Aceito em: 26/09/2014. 
A criptosporidiose é uma zoonose de ampla distribuição mundial causada por protozoários pertencentes ao gênero Cryptosporidium, que acomete várias espécies de vertebrados (Diaz De Ramirez et al., 2002; Fayer, 2010).

Embora as informações sobre a ocorrência de Cryptosporidium em algumas espécies animais sejam escassas, os estudos já existentes no Brasil demonstram que a criptosporidiose apresenta características clínicas e epidemiológicas semelhantes àquelas descritas em outros países, particularmente em relação aos ruminantes, cavalos, cães e gatos. As espécies e genótipos encontrados em aves e mamíferos (incluindo seres humanos) já foram descritos em outros países (Meireles, 2010).

$\mathrm{Na}$ literatura, os resultados dos inquéritos epidemiológicos são muito variáveis e a morbidade pode variar de 10 a $85 \%$, com uma ocorrência média de $25 \%$ em bezerros (Kirkpatrick, 1985). A presença de Cryptosporidium em animais diarreicos é relatada por Dubey et al. (1992), que consideraram esse coccídeo como um dos mais importantes enteropatógenos de ruminantes domésticos. Oliveira FilHo et al. (2007), no Brasil, apontaram esse agente como o segundo enteropatógeno mais frequentemente encontrado nos bezerros de corte apresentando diarreia.

Pesquisas demonstraram uma maior incidência de Cryptosporidium em fezes de búfalos com sinais clínicos de diarreia (Galiero et al., 1994). Contudo, outros estudos realizados para avaliar a etiologia e dinâmica da infecção por enteropatógenos causadores de diarreia em bezerros búfalos demonstraram maior ocorrência de $C$. parvum em animais sem diarreia, como no estudo de Ribeiro et al. (2000) que, ao descreveram 9,43\% (10/106) de amostras positivas para o agente, observaram que 60\% (6/10) dessas amostras eram de animais sem diarreia. Da mesma forma, Rinaldi et al. (2007) constataram que $24,44 \%$ (22/90) das fazendas pesquisadas tinham pelo menos um bezerro positivo para $C$. parvum, independentemente de apresentarem diarreia ou não. EDERLI et al. (2004), estudando 211 amostras fecais de bezerros mestiços, 111 fêmeas e 100 machos, com idade variando de 4 dias a 12 meses, também não observaram correlação entre a ocorrência de diarreia e infecçâo por Cryptosporidium.

Dentre os bubalinos, a infecçáo por Cryptosporidium tem sido pesquisada em alguns países buscando determinar sua importância como agente etiológico da síndrome diarreica entre bubalinos jovens e imunossuprimidos. Apesar da vasta pesquisa e importância desse patógeno causador de grandes perdas econômicas, há uma carência de estudo em animais de produção na Amazônia Oriental.

Nesse sentido, objetivou-se pesquisar a ocorrência de Cryptosporidium spp. em bezerros bubalinos no estado do Pará e correlacionar sua ocorrência com a faixa etária e a presença de sinais clínicos da diarreia nos animais estudados.

Amostras fecais foram coletadas de 253 bezerros com até 6 meses de idade com ou sem histórico de diarreia, provenientes de 7 propriedades rurais, localizadas nos municípios de Soure ( $0^{\circ} 23^{\prime} 32.27^{\prime \prime} S$, 48 $\left.38^{\circ} 11.57^{\prime \prime} \mathrm{O}\right)$, na Mesorregião da Ilha de Marajó, Belém (1²6’38.74”, 48'29'47.96”O), na Mesorregiấo Metropolitana de Belém, São Caetano de Odivelas (056'26.43”'S, 4801'13.70”O), Nova Timboteua ( $\left.1^{\circ} 12^{\prime} 07.31^{\prime \prime} S, \quad 47^{\circ} 24^{\prime} 32.00^{\prime \prime} \mathrm{O}\right)$ e Mojú ( $\left.{ }^{\circ} 53^{\prime} 01.14^{\prime \prime} S, 48^{\circ} 45^{\prime} 55.82^{\prime \prime O}\right)$, na Mesorregiáo do Nordeste Paraense, e Rondon do Pará ( $4^{\circ} 46^{\prime} 40.69^{\prime \prime}$, 4804'02.8”'O), na Mesorregião do Sudeste Paraense.

Os animais foram separados em três grupos de acordo com a idade: menos de dois meses, dois a quatro meses incompletos e quatro a seis meses. As fezes foram obtidas diretamente do reto, mediante estimulação manual. Em seguida, foram inspecionadas e classificadas como diarreicas ou não diarreicas, de acordo com a consistência e o aspecto. Foram consideradas como diarreicas as de consistência pastosa, aquosa e liquefeitas, e não diarreicas aquelas de consistência firme. Após a coleta foram acondicionadas em frascos tipo falcon, identificadas e transportadas em caixa isotérmica contendo gelo reciclável para processamento e análise no Laboratório de Parasitologia do Centro Nacional de Primatas (CENP) do Instituto Evandro Chagas, Ananindeua, estado do Pará.

No processamento laboratorial, as fezes frescas, sem conservantes, foram submetidas ao processo de concentraçáo pelo formol-éter modificado. Cerca de 1 a $2 \mathrm{~g}$ de fezes foram homogeneizados em $10,0 \mathrm{~mL}$ de solução de formalina a $10 \%$ e filtradas em tamis de plástico descartável para facilitar a visualizaçáo dos oocistos. Uma parte equivalente a $4,0 \mathrm{~mL}$ desse filtrado foi colocada em tubos tipo falcon, acrescida de 2,0 mL de éter e centrifugada a $2000 \mathrm{G}$ por 10 minutos. Com o sobrenadante descartado, duas gotas do sedimento foram utilizadas na confecçâo de esfregaços em lâmina de vidro e secos à temperatura ambiente.

Após a fixação em álcool metílico por 30 segundos, os esfregaços foram submetidos ao método de coloração pela fucsina carbólica (corante de Kinyoun) por 15 minutos, descoloração pelo álcool-ácido sulfúrico por 2 minutos, lavagem rápida com água destilada e contra-coloração pelo azul de metileno por 2 minutos. A observaçáo dos oocistos de Cryptosporidium spp. foi realizada em microscópio óptico em objetivas de 40 e $100 \mathrm{x}$.

Para determinar a relaçáo entre idade do animal e presença de Cryptosporidium spp. foi aplicada a estatística teste $(\mathrm{G})$.

Das 253 amostras fecais de bezerros bubalinos analisadas, 2,37\% (6/253) eram positivas para oocistos de Cryptosporidium (Tabela 1; Fig. 1). Índices aproximados foram descritos por Wade et al. (2000), que examinaram 2.943 amostras de fezes de bovinos leiteiros jovens e adultos e registraram uma prevalência de 2,4\% (27/1.135) em bezerros com menos de 6 meses de idade para o C. parvum. Prevalências moderadas foram descritas por 
Tabela 1. Ocorrência de Cryptosporidium spp. em amostras de fezes de bezerros bubalinos de acordo com o grupo etário estudado, no estado do Pará.

\begin{tabular}{|c|c|c|c|c|c|c|c|c|}
\hline \multirow{3}{*}{ Amostras } & \multicolumn{6}{|c|}{ Grupos etários } & \multirow{2}{*}{\multicolumn{2}{|c|}{ Total }} \\
\hline & \multicolumn{2}{|c|}{$<2$ meses } & \multicolumn{2}{|c|}{ 2- 4 meses } & \multicolumn{2}{|c|}{ 4- 6 meses } & & \\
\hline & $\mathbf{n}$ & $\%$ & $n$ & $\%$ & $\mathbf{n}$ & $\%$ & $\mathrm{n}$ & $\%$ \\
\hline Positivas & 2 & 2,11 & 3 & 2,54 & 1 & 2,5 & 6 & 2,37 \\
\hline Negativas & 93 & 97,89 & 115 & 97,46 & 39 & 97,5 & 247 & 97,63 \\
\hline Total & 95 & 100 & 118 & 100 & 40 & 100 & 253 & 100 \\
\hline
\end{tabular}

$G=0,0475 ; p=0,976$.

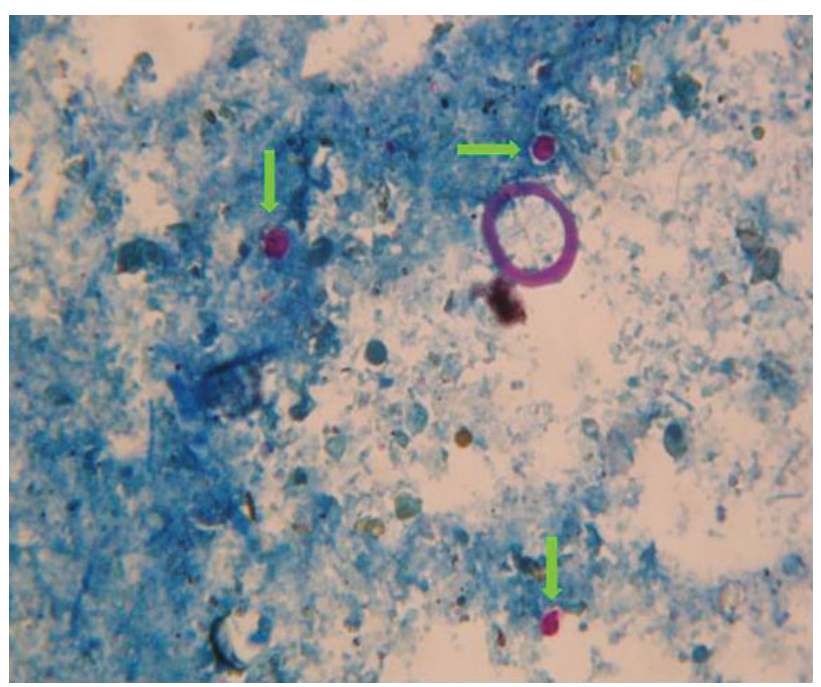

Figura 1. Oocistos de Cryptosporidium spp. em amostras de fezes de bezerro bufalino (setas). Técnica de coloração de Kinyoun (aumento de $100 \mathrm{x}$ ).

Amer et al. (2013), que observaram 9,5\% (17/179) de animais positivos, e por Cacciò et al. (2007), que descreveram uma taxa de 14,0\% (8/57) de bezerros búfalos italianos positivos. Já Bhat et al. (2012) e Feng et al. (2012) registraram taxas de infecção iguais a 38,3\% (62/162) e 37,04\% (30/81) em bezerros búfalos na Índia e Nepal. Essas discrepâncias nas taxas de infecçáo relatadas podem ser atribuídas a diferenças na idade e raça dos animais, época da coleta das amostras, condiçôes edafoclimáticas e manejos e sistemas de criação dos animais (AMER et al., 2013). A falta de deteç̧ão de Cryptosporidium em bubalinos adultos está de acordo com resultados de estudos anteriores em outros países. De 616 e 525 búfalos examinados em Cuba e Egito, respectivamente, oocistos foram detectados apenas em bezerros (АвоU-Eisha, 1994; Rodriguez Diego et al., 1991).

Das amostras estudadas, as positivas foram provenientes de animais sem sinais clínicos de diarreia, estando compatível com os resultados encontrados por Mota et al. (2000), que náo encontraram oocistos de Cryptosporidium em nenhuma das 20 amostras diarreicas analisadas, e menores do que aqueles descritos por Ederli et al. (2004), que observaram $11,76 \%(2 / 17)$ dos animais com sinais clínicos de diarreia positivos na pesquisa de oocistos de Cryptosporidium, enquanto que naqueles assintomáticos, a infecção foi de 46,40\% (90/194). Esses resultados diferem daqueles obtidos por Bhat et al. (2012), em que oocistos de Cryptosporidium foram diagnosticados em 49\% (37/75) das amostras de animais diarreicos e $28,73 \%$ (25/87) dos animais assintomáticos.

Assim sendo, os dados do presente estudo corroboram a assertiva de Ribeiro et al. (2000) ao afirmarem que a ocorrência de agentes parasitários em ambos os bezerros com diarreia e assintomáticos, a despeito de não ter significância estatística nos animais com diarreia ( $p>0,05)$, demonstra a importância de todos os bezerros como fonte potencial de infecção.

$\mathrm{Na}$ presente pesquisa a infecção ocorreu nas três faixas etárias, com índices aproximadamente iguais (em torno de 2\%) (Tabela 1). SouzA; Lopes (1995) também detectaram o Cryptosporidium spp. tanto em animais com menos 30 dias como naqueles com idade entre 30 e 90 dias. Em bezerros com até 30 dias de idade, a maior taxa de excreçâo $(14,5 \%)$ foi observada nos animais com até 14 dias de idade e a menor nos animais mais velhos (22 a 30 dias) (Feitosa et al., 2004). Entretanto, Surumay-Vilchez; Sandoval (2000) encontraram animais positivos somente no grupo etário com mais de quatro semanas.

Essas divergências podem ser justificadas em função das distintas práticas de manejo adotadas na criação dos animais que, segundo OrTOLANI (1988), envolvem desde aspectos de densidade populacional, estratificaçáo dos animais por idade, condiçôes de higiene e virulência dos micro-organismos associados, que podem influenciar na ocorrência da infecção. Para o autor, a maior taxa de infecção em animais de até 30 dias de idade é atribuída à baixa imunidade em animais recém-nascidos e à facilidade de contaminação pelas mamadeiras ou baldes de alimentação. Ainda, quanto piores forem as condiçóes sanitárias encontradas no ambiente, maior será o risco de contágio e aparecimento de doenças entre bezerros neonatos.

Das sete propriedades pesquisadas, três apresentaram animais positivos para oocistos de Cryptosporidium spp. com maior percentual naquela que utilizava o sistema de confinamento $(15,38 \% ; 2 / 13)$, estando em conformidade com o 
proposto por OrTOLANI (1988), que atribuiu um percentual mais elevado para a presença de Cryptosporidium spp. ao confinamento associado à condiçáo sanitária precária e às práticas de manejo deficientes.
A infecção por Cryptosporidium spp. em bezerros bubalinos com até seis meses de idade no estado do Pará foi diagnosticada apenas em animais não diarreicos, com baixa ocorrência, independente da faixa etária.

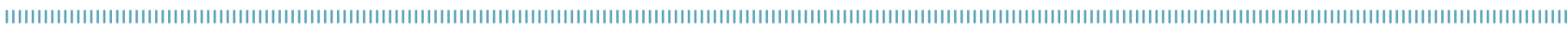

\section{REFERÊNCIAS}

ABOU-EISHA, A.M. Cryptosporidial infection in man and farm animals in Ismailia Governorate. Veterinary Medical Journal Giza, v.42, n.2, p.107-111, 1994.

AMER, S.; ZIDAN, S.; FENG, Y.; ADAMU, H., LI, N.; XIAO, L. Identity and public health potential of Cryptosporidium spp. in water buffalo calves in Egypt. Veterinary Parasitology, v.191, n. 1-2, p.123-127, 2013.

BHAT, S.; JUYAL, P.; SINGLA, L. Prevalence of cryptosporidiosis in neonatal buffalo calves in Ludhiana District of Punjab, India. Asian Journal of Animal and Veterinary Advances, v.7, n.6, p.512-520, 2012.

CACCIÒ, S.M.; RINALDI, L.; CRINGOLI, G.; CONDOLEO, R.; POZIO, E. Molecular identification of Cryptosporidium parvum and Giardia duodenalis in the Italian water buffalo (Bubalus bubalis). Veterinary Parasitology, v. 150, n. 1-2, p. 146-149, 2007.

DIAZ DE RAMÍRES, A.; RAMÍREZ-IGLESIA, L.N.; GODOY DE PLAZA, R.M.; ROMÁN BRAVO, R. Excreción de ooquistes de Cryptosporidium spp. durante el postparto, en vacas mestizas de doble propósito. Revista Científica, v. 12, n.2, p.614-616, 2002.

DUBEY, J.P.; FAYER, R.; RAO, J.R. Cryptosporidial oocyst in faeces of water buffalo and zebu calves in India. Journal of Veterinary, v.6, n.1, p.55-6, 1992.

EDERLI, B.B.; CARVALHO, C.B.; SALES, L.G. Ocorrência da infecção por Cryptosporidium em bezerros na Microrregião de Campos dos Goytacazes no Norte do Estado do Rio de Janeiro, Brasil. Revista Brasileira de Parasitologia Veterinária, v.13, n.2, p.45-48, 2004.

FAYER, R. Taxonomy and species delimitation in Cryptosporidium. Experimental Parasitoigy, v.124, n.1, p.90-97, 2010.

FEITOSA, F.L.F.; SHIMAMURA, G.M.; ROBERTO, T.; MEIRELES, M.V.; NUNES, C.M.; CIARLINI, P.C.; BORGES, A.S. Prevalência de criptosporidiose em bezerros na região de Araçatuba, Estado de São Paulo. Ciência Rural, v.34, n. 1, p.189-193, 2004.

FENG, Y.; KARNA, S.R.; DEAREN, T.K.; SINGH, D.K.; ADHIKARI, L.N.; SHRESTHA, A.; XIAO, L. Common occurrence of a unique Cryptosporidium ryanae variant in zebu cattle and water buffaloes in the buffer zone of the Chitwan National Park, Nepal. Veterinary Parasitology, v.185, n.2-4, p.309-314, 2012.

GALIERO, G.; CONSALVO, F.; CARULLO, M. La criptosporidiosi nei vitelli bufalini: un aggiornamento. Selezione Veterinaria, v.35, p.449-453, 1994.

KIRKPATRICK, C.E. Cryptoporidium infection as cause of diarrhea. Veterinary Clinics of North America: Food Animal Practice, v.1, n.3, p.515-528, 1985.
MEIRELES, M.V. Cryptosporidium infection in Brazil: implications for veterinary medicine and public health. Revista Brasileira de Parasitologia Veterinária, v.19, n.4, p.197-204, 2010.

MOTA, R.A.; SILVA, K.P.C.; RIBEIRO, T.C.F.; RAMOS, G.A.B.; LIMA, E.T.; SILVA, L.B.G.; ZÜNIGA, C.E.A. Eficácia do Nuflor no tratamento de diarréias em bezerros e leitões. A Hora Veterinária, v.20, n.118, p.21-24, 2000.

OLIVEIRA FILHO, J.P.; SILVA, D.P.G.; PACHECO, M.D.; MASCARINI, L.M.; RIBEIRO, M.G.; ALFIERI, A.A.; ALFIERI, A.F.; STIPP, D.T.; BARROS, B.J.P.; BORGES, A.S. Diarréia em bezerros da raça Nelore criados extensivamente: estudo clínico e etiológico. Pesquisa Veterinária Brasileira, v.27, n.10, p.419-424, 2007.

ORTOLANI, E.L. Padronização da tecnica de Ziehl-Neelsen para pesquisa de oocistos de Cryptosporidium: estudo de alguns aspectos epidemiológicos de criptosporidiose em bezerros de rebanhos leiteiros no estado de São Paulo. 1988. 85f. Tese (Doutorado) - Universidade de São Paulo, São Paulo, 1988

RIBEIRO, M.G.; LANGONI, H. JEREZ, J.A.; LEITE, D.S.; FERREIRA, F.; GENNARI, S.M. Identification of enteropathogens from buffalo calves with and withoug diarrhoea in the Ribeira Valley, State of São Paulo, Brasil. Brazilian Journal of Veterinary Research and Animals Science, v.37, n.2, p.159-165, 2000.

RINALDI, L.; MUSELLA, V.; CONDOLEO, R.; SARALLI, G.; VENEZIANO, V.; BRUNI, G.; CONDOLEO, R.U.; CRINGOLI, G. Giardia and Cryptosporidium in water buffaloes (Bubalus bubalis). Parasitology Research, v.100, n.5, p.11131118,2007

RODRIGUEZ DIEGO, J.; ABREU, J.R.; PÉREZ, E.; ROQUE, E.; CARTAS, O. Presence of Cryptosporidium in buffaloes (Bubalus bubalis) in Cuba. Revista Salud Animal, v.13, p.78-80, 1991.

SOUZA, J.C.P.; LOPES, C.W.G. Criptosporidiose em bezerros de rebanhos da bacia leiteira Sul-Fluminense, Estado do Rio de Janeiro. Revista Brasileira de Parasitologia Veterinária, v.4, n.1, p.33-36, 1995.

SURUMAY-VILCHEZ, Q.; SANDOVAL, Y. Cryptosporidium parvum en búfalos de una finca del município Mara, Estado ZuliaVenezuela. Veterinária Tropical, v.25, n.2, p.285-290, 2000.

WADE, S.E.; MOHAMMED, H.O.; SCHAAF, S.L. Prevalence of Giardia sp. Cryptosporidium parvum and Cryptosporidium andersoni (syn. C. muris) [correction of cryptosporidium parvum and Cryptosporidium muris-C. andersoni)] in 109 dairy herds in five counties of southeastern New York. Veterinary Parasitology, v.93, n. 1, p. 1-11, 2000 